\title{
Optimisation potentielle de la maintenance d'EnBW : bonnes pratiques
}

par Dr. ZIMMER, H. JANISCH, EnBW Kraftwerke AG, Philippsburg

\section{Introduction}

La gestion sûre et économique des centrales nucléaires est et restera à l'avenir l'objectif prioritaire de leurs exploitants. Avec la libéralisation du marché de l'électricité, la gestion économique a gagné en importance par rapport au passé.

Déjà par le passé, les exploitants ont cherché à réduire leurs coûts de production. On peut notamment citer l'optimisation de la production annuelle d'électricité ou encore le remplacement des corps haute pression et basse pression des turbines, I'augmentation du rendement thermique ainsi que la disponibilité renforcée grâce à la durée plus courte des arrêts de tranche. Etant donné qu'il y a très peu d'arrêts non programmés en dehors des arrêts de tranche, l'impact en termes de capacité sur le dénominateur des coûts spécifiques de production n'a pratiquement pas d'incidence.

Ainsi, c'est le numérateur, c'est-à-dire le vecteur coût, qui vient en tout premier plan en termes de programme d'optimisation à engager. Chez EnBW-Kraftwerksgesellschaft, la part de la maintenance dans le coût de production de l'électricité est d'environ $19 \%$. Elle représente la partie la plus importante des coûts d'exploitation.

- La maintenance permet, sur une installation technique, d'obtenir une performance dans une plage de tolérance déterminée. Dans une centrale, on peut - de façon simplifiée - diviser la maintenance en trois domaines : entretien, contrôles - visites et réparations

On peut également diviser la maintenance en maintenance préventive, conditionnelle et curative.

Lorsque I'on s'attache au concept d'optimisation de la maintenance, il faut envisager l'ensemble du processus de maintenance avec les différentes entités qui lui viennent en appui. II faut donc également considérer la partie logistique, par exemple le processus d'achats, ainsi que les processus internes à l'entreprise. En outre, il faut tenir compte d'autres aspects comme la maintenance « multisites ". Il convient d'identifier et d'utiliser les synergies qui découlent des rapprochements d'entreprises. L'approche purement commerciale des achats n'a pas besoin d'être, ici, approfondie.

\section{Passage de la maintenance préventive à la maintenance conditionnelle}

La stratégie des exploitants est de faire fonctionner leurs installations de façon à ce qu'une défaillance soudaine de composant n'ait pas d'impact pénalisant sur les systèmes. Pour que I'on puisse appliquer ce principe de conduite vraisemblablement optimal tout au long de la durée de vie d'une centrale, il convient d'opter pour une gestion optimisée et une stratégie de maintenance mûrement réfléchie. Ceci est d'autant plus vrai lorsque I'on songe qu'au cours d'une année, près de 7000 interventions récurrentes de maintenance ont lieu sur chaque tranche dans le cadre de la maintenance préventive et environ 4000 essais périodiques. En moyenne, près de 1 400 fiches d'écart par tranche sont traitées chaque année.

Comparés à leurs homologues dans le monde, les exploitants de centrales nucléaires allemandes enregistrent d'excellents résultats en termes de disponibilité et de fiabilité de leurs installations.

Dans le cas de la maintenance curative, le système ou le composant est exploité jusqu'au moment où il tombe en panne ou est endommagé. On ne peut opter pour cette stratégie que lorsqu'il n'y a pas d'incidence sur les aspects sûreté, lorsqu'il n'en résulte pas des contraintes d'exploitation imprévues et que le remplacement d'un composant endommagé est plus économique que sa réparation.

Etant donné que la sûreté est la priorité $n^{\circ} 1$, on a décidé, par le passé, d'établir des fréquences fixes pour la réalisation des interventions de maintenance. Comme au départ l'expérience en ce domaine n'était pas très développée, ces fréquences étaient très conservatoires, ce qui, du point de vue de la sûreté, ne présente pas de risque mais est par ailleurs coûteux. La résistance des composants à l'usure restait en grande partie intacte.

La maintenance conditionnelle implique que les composants ou le système peuvent être surveillés - en permanence ou non - grâce à des outils de diagnostic capables de détecter et de suivre la formation d'un défaut bien avant apparition de la défaillance La pratique optimale de 
la maintenance conditionnelle ne comporte pas de risque pour l'exploitation des sites et représente donc la méthode de maintenance la plus économique. Entre temps, les systèmes de diagnostic et les stratégies les plus variés existent et sont utilisés.

\section{Utilisation des résultats des analyses probabilistes de sûreté (PSA) pour la maintenance}

Dans le cadre de l'évaluation périodique de sûreté (PSU en allemand), des analyses probabilistes de sûreté ont été effectuées dans toutes les centrales nucléaires allemandes. L'accent a été mis sur les risques dans les analyses PSA de niveau 1 et le contrôle de l'équilibre dans les systèmes de sûreté. Jusqu'à présent, il n'y a pas encore eu d'analyse poussée des résultats des PSA par rapport aux exigences de la maintenance mais on pourrait envisager l'optimisation potentielle de la maintenance en ciblant cet aspect. En d'autres termes, si pour les analyses PSA initiales on mettait l'accent sur l'évaluation de sûreté des systèmes importants nécessaires pour atteindre les objectifs de protection (comme par exemple le refroidissement du cœur ou l'évacuation de la chaleur résiduelle en tout sûreté) on pourrait rechercher, dans le cadre d'une utilisation élargie des analyses probabilistes de sûreté, les systèmes du circuit secondaire importants pour la disponibilité en envisageant comment optimiser les interventions de maintenance - pour autant que ni la disponibilité, ni la sûreté de l'ensemble des installations, ne s'en ressentent. A l'heure actuelle, on procède à des analyses probabilistes de disponibilité de ce type dans certaines centrales nucléaires scandinaves. Nous allons suivre ces développements avec intérêt et envisageons d'ores et déjà d'effectuer des analyses similaires une fois que les travaux en cours seront achevés et analysés.

\section{Organisation pour la mise en œuvre et le déroulement}

La modification de stratégies avancées de maintenance ne pourra durablement réussir que si l'organisation du travail et la gestion du personnel sont compatibles avec ces nouvelles stratégies. L'organisation de la maintenance conditionnelle cible la fiabilité de systèmes complets et non pas le maintien préventif de composants ou de pièces spécifiques. La mise en œuvre de la maintenance conditionnelle exige donc une coopération transverse renforcée de tous les acteurs de la maintenance. L'organisation de l'entreprise conçue et orientée autour des systèmes et des process permet de s'assurer que les composants sont entretenus non pas selon des critères individuels ou par spécialité, mais plutôt pour la fiabilité nécessaire des systèmes. Outre l'organisation, il convient également de former le personnel à cette évolution de la stratégie de la maintenance. Alors que pour la maintenance préventive, il s'agit essentiellement de mettre en œuvre un planning donné de maintenance, la maintenance conditionnelle nécessite une responsibilisation et des compétences techniques accrues de la part des acteurs de la maintenance. II convient d'orienter le management et la formation de ces acteurs par rapport à ces aspects.
Pour conforter le bon déroulement de la maintenance optimisée, il est recommandé d'adopter une organisation simple au nombre d'interfaces limité. Ceci est d'autant plus important dans les périodes où les ressources humaines sont limitées.

Pour instaurer une bonne rigueur d'exécution, il faut si possible confier l'ensemble des interventions de maintenance routinière à un seul service, alors que les modifications et les projets - c'est-à-dire les missions des ingénieurs - sont traitées dans un autre service. Ce service est ainsi entièrement déchargé de la routine quotidienne et peut remplir ses tâches de façon plus efficace.

Par interventions routinières de maintenance, on entend les interventions figurant dans les programmes d'essais, les plannings de maintenance ou de visite et qui se répètent de façon régulière. En outre, par maintenance de routine, on entend également l'élimination des défaillances mineures.

\section{Outils informatiques}

L'exploitation et la maintenance ne peuvent être économiques que s'il y a suffisamment de transparence de la part de l'entité générant les coûts (donneur d'ordre), répartis selon les parties de systèmes et les composants de tranche et en fonction des intervenants. C'est pourquoi il est nécessaire de disposer d'informations nettement plus détaillées et circonstanciées qu'aujourd'hui.

Dans la plupart des centrales, on a, au cours des années passées, introduit différents systèmes de gestion des entreprises. Parmi les systèmes les plus répandus, on compte notamment BFS de Siemens et IBFS de l'entreprise GIS. Le degré d'implantation de ces systèmes varie d'un site à l'autre, en fonction de l'environnement organisationnel. Ils sont principalement utilisés pour venir en appui à la gestion de l'entreprise dans le domaine technique mais ils n'ont encore que trop peu de liens avec les magasins. En outre, SAP-R3 (domaine commercial) a été installé entre temps dans de nombreuses centrales ou est actuellement en cours d'installation. Ce système à double voie ne permet pas d'obtenir aisément et en temps voulu les informations nécessaires sur les coûts et de plus, il complique le travail quotidien car il faut penser et travailler dans deux logiques différentes.

\section{Recours aux prestataires de maintenance}

A l'heure actuelle, près de la moitié des prestations de maintenance sont réalisées par du personnel externe. Celui-ci se répartit en 1/3 de prestataires permanents et $2 / 3$ de prestataires de travaux et services. Compte tenu des sureffectifs internes liés aux réorganisations et fermetures d'installations et aux travaux confiés à ces équipes, on peut se demander ce qu'il reste à sous-traiter !

Voici la réponse : on a besoin des prestataires et dans de nombreux domaines, ils sont même irremplaçables ! Cependant, les entreprises prestataires doivent s'adapter à la nouvelle donne. C'est la raison pour laquelle on leur demande des stratégies visant à réduire les coûts tout leur assurant un chiffre d'affaire suffisant, pour leur permettre de fournir une prestation de qualité. 
L'utilisation de prestataires permet de réduire les coûts de production parce que :

- le nombre d'employés nécessaires est limité

- le maintien de personnel en réserve pour des travaux périodiques est ainsi évité,

- le recours aux experts disponibles des entreprises prestataires est ainsi optimisé,

- I'utilisation de prestataires spécialistes disponibles dans plusieurs centrales est rationnelle,

- les locaux et installations techniques des sociétés prestataires intervenant à plusieurs endroits sont plus économiques que les installations propres rarement utilisées.

Pendant les arrêts de tranche, les activités ne peuvent pas être réalisées uniquement par les agents d'un site. On peut donc optimiser l'utilisation de prestataires en période d'arrêt en accordant des lots d'interventions qui représentent un volume et un déroulement dans le temps tels que les déficits d'activités peuvent être compensés par des interventions dans d'autres domaines ou au contraire, que les goulots d'étranglement peuvent être évités grâce à la mise en place d'employés d'autres domaines.

\section{Conclusions}

Du point de vue de l'exploitant, l'attribution de commandes par lot ne doit pas provoquer l'élimination de toute concurrence. II faut par principe maintenir et conserver intact un marché sur lequel il y a plusieurs offres possibles.

En fait, il faut considérer les centrales nucléaires comme des entreprises de maintenance produisant de l'électrici- té. A l'avenir, il nous faudra choisir une stratégie de maintenance pour nos installations qui prévoira des interventions optimisées pour chaque composant de tranche et en tout état de cause, si possible à un faible coût. Ce n'est que de cette façon que nous pourrons garantir au marché un approvisionnement énergétique sûr au prix le plus bas possible tout en nous assurant un bénéfice suffisant sur chaque période de fonctionnement. Sur le marché libéralisé de l'électricité et dans les conditions de concurrence prévalant à l'heure actuelle, ce sont les prix offerts (à savoir le coût marginal en $\mathrm{Pf} / \mathrm{kWh}$ ) découlant de la production qui déterminent si un acteur du marché pourra continuer à vendre de l'électricité avec sa capacité de production. Dans une seconde étape, ce sont les marges sur les coûts variables obtenues grâce à l'exploitation des tranches qui décideront si l'ensemble des coûts fixes peut être couvert. Si ce n'est pas le cas, la centrale en question devra se retirer du marché.

A l'avenir, on exigera également des exploitants qu'ils aient les marges nécessaires pour pouvoir maintenir le niveau de sûreté de leurs centrales et intégrer les progrès de la technique. Dans le contexte concurrentiel actuel, c'est aujourd'hui et non pas demain qu'il faudra plus que jamais et davantage faire la part des choses entre les coûts et les profits dans la mise en œuvre d'interventions de maintenance et placer cet aspect en priorité.

C'est pourquoi les procédés entièrement homogènes d'évaluation de la sûreté des installations figurent au premier plan de nos préoccupations prioritaires. Nous ferons bon usage de toutes les possibilités existantes pour renforcer plus encore l'efficacité de notre processus de maintenance, en commençant par l'utilisation de systèmes de diagnostic intelligents jusqu'à la mise en œuvre d'analyses probabilistes de sûreté pour nous permettre d'évaluer nos stratégies de maintenance. 